\title{
Dynamics Control of the Complex Systems via Nondifferentiability
}

\author{
Carmen Nejneru, ${ }^{1}$ Anca Nicuţă, ${ }^{2}$ Boris Constantin, ${ }^{1}$ Liliana Rozemarie Manea, ${ }^{3}$ \\ Mirela Teodorescu, ${ }^{4}$ and Maricel Agop ${ }^{5,6}$ \\ ${ }^{1}$ Faculty of Materials Science and Engineering, "Gheorghe Asachi” Technical University of Iaşi, 41 D. Mangeron Boulevard, \\ 700050 Iași, Romania \\ ${ }^{2}$ Faculty of Civil Engineering and Building Services, "Gheorghe Asachi” Technical University of Iaşi, 1 D. Mangeron Boulevard, \\ 700050 Iaşi, Romania \\ ${ }^{3}$ Faculty of Textile, Leather Engineering and Industrial Management, "Gheorghe Asachi” Technical University of Iaşi, \\ Dimitrie Mangeron 29, 700050 Iaşi, Romania \\ ${ }^{4}$ Institute of Macromolecular Chemistry Petru Poni Iaşi, Aleea Grigore Ghica Voda, No. 41A, 700487 Iaşi, Romania \\ ${ }^{5}$ Physics Department, Faculty of Machine Manufacturing and Industrial Management, "Gheorghe Asachi” Technical University of Iaşi, \\ Professor Dr. Docent Dimitrie Mangeron Road, No. 59A, 700050 Iaşi, Romania \\ ${ }^{6}$ Lasers, Atoms and Molecules Physics Laboratory, University of Science and Technology, Villeneuve d'Ascq, 59655 Lille, France
}

Correspondence should be addressed to Maricel Agop; m.agop@yahoo.com

Received 29 May 2013; Accepted 12 July 2013

Academic Editor: Zhiwei Gao

Copyright (C) 2013 Carmen Nejneru et al. This is an open access article distributed under the Creative Commons Attribution License, which permits unrestricted use, distribution, and reproduction in any medium, provided the original work is properly cited.

\begin{abstract}
A new topic in the analyses of complex systems dynamics, considering that the movements of complex system entities take place on continuum but nondifferentiable curves, is proposed. In this way, some properties of complex systems (barotropic-type behaviour, self-similarity behaviour, chaoticity through turbulence and stochasticization, etc.) are controlled through nondifferentiability of motion curves. These behaviours can simulate the standard properties of the complex systems (emergence, self-organization, adaptability, etc.).
\end{abstract}

\section{Introduction}

Complex systems are very large interdisciplinary research topics that have been intensively studied, particularly since the 1980s, by means of a combination of basic theory, derived especially from physics and computer simulation. Such kind of systems is composed of many interacting elemental units that were called "agents." Examples of complex systems can be found in human societies, the brain, internet, ecosystems, biological evolution, stock markets, economies, and many others [1-4].

The way in which such a system manifests cannot be predicted only by the behaviour of individual elements or by adding their behavior but is determined by the manner in which the elements relate to influence global behaviour. Among the most significant properties of complex systems are emergence, self-organization, adaptability, and so forth [5-9].

The emergence of a complex system can be represented by that state of the whole which does not include the sum of its elements.

Self-organization is another characteristic of complex systems. Examples of organization that manifests in nature are found from cells to organisms, ecosystems, and also planets, stars, and galaxies [5-9].

Another characteristic of complex systems is adaptability. This occurs when the system changes in response to some information. Continuously oscillating between equilibrium and disorder, complex systems are not "rigid." Any modification induced at microlevel generates a series of fluctuations, exploring new states of self-organization [5-9]. 
An example of a complex system is represented by polymers. Their forms present a multitude of organizations starting from simple, linear chains of identical structural units to very complex chains consisting of sequences of amino acids that form the building blocks of living things. Probably one of the most intriguing complex systems in nature is DNA, which creates cells by means of a simple but very elegant language. It is responsible for the remarkable way in which individual cells organize into complex systems like organs, and these organs form even more complex systems like organisms. The study of complex systems can offer a glimpse about the realistic dynamics of polymers, solving difficult problems as protein folding [6-10].

Correspondingly, the theoretical models that describe the complex systems dynamics become sophisticated and ambiguous too [6-10]. However the situation can be standardized taking into account that the complexity of interaction process imposes various temporal resolution scales, and the pattern evolution imposes different degrees of freedom.

In order to develop a new theoretical model we must admit that the complex systems that display chaotic behaviour are known to acquire self-similarity (space-time structures seem to appear) in association with strong fluctuations at all possible space-time scales [6-8]. Then, for temporal scales that are large with respect to the inverse of the highest Lyapunov exponent, the deterministic trajectories are replaced by a collection of potential trajectories and the concept of definite positions by that of probability density [11-14]. One of the most interesting examples is the collisions processes in plasma discharge as a complex system, where the dynamics of the particles can be described by Levi-type movements [15]: between two successive collisions, the particle trajectory is a straight line, with the trajectory becoming nondifferentiable in the impact point, which implies that there are left and right derivatives in this point. We note that the Brownian-type motion is a particular case of Levi-type motion [16].

Since the nondifferentiability appears as a universal property of the complex systems, it is necessary to construct a nondifferentiable physics. In such conjecture, by considering that the complexity of the interactions processes is replaced by nondifferentiability, it is no longer necessary to use the whole classical "arsenal" of quantities from the standard physics (differentiable physics) [12-14, 17, 18].

This topic was developed in [12-14, 19-23] using the scale relativity theory (SRT) [12-14]. In the framework of SRT we assume that the movements of complex system entities take place on continuous but nondifferentiable curves (fractal curves) so that all physical phenomena involved in the dynamics depend not only on the space-time coordinates but also on the space-time scales resolution. From such a perspective, the physical quantities that describe the dynamics of complex systems may be considered fractal functions [12-14]. Moreover, the entities of the complex system may be reduced to and identified with their own trajectories, so that the complex system will behave as a special interactionless "fluid" by means of its geodesics in a nondifferentiable (fractal) space.
In the present paper, we propose a new topic to analyse the complex systems dynamics using SRT. Considering that the entities of the complex system are moving on continuous but nondifferentiable curves, we show that the control of different behaviours of these systems implies nondifferentiability.

\section{Geodesics Equations}

Considering that the dynamics of the complex system entities take place on continuous but nondifferentiable curves, that is, fractal curves (e.g., the Koch curve, the Peano curve, or the Weierstrass curve $[11-14,17,18]$ ), they are given by the fractal operator $\widehat{d} / d t$ (for details see Appendices A and $\mathrm{B}$ ):

$$
\frac{\widehat{d}}{d t}=\frac{\partial}{\partial t}+\widehat{\mathbf{V}} \cdot \nabla-i \frac{\lambda^{2}}{\tau}\left(\frac{d t}{\tau}\right)^{\left(2 / D_{F}\right)-1} \Delta,
$$

where

$$
\widehat{\mathbf{V}}=\mathbf{V}_{D}-i \mathbf{V}_{F}
$$

is the complex velocity, $\mathbf{V}_{D}$ is the differentiable and resolution scale independent velocity, $\mathbf{V}_{F}$ is the nondifferentiable and resolution scale dependent velocity, $\widehat{\mathbf{V}} \cdot \nabla$ is the convective term,

$$
\begin{aligned}
\left(\frac{\lambda^{2}}{\tau}\right)\left(\frac{d t}{\tau}\right)^{\left(2 / D_{F}\right)-1} \Delta= & \left(\frac{\lambda^{2}}{\tau}\right)\left(\frac{d t}{\tau}\right)^{\left(2 / D_{F}\right)-1} \\
& \times\left[\left(\frac{\partial^{2}}{\partial x^{2}}\right)+\left(\frac{\partial^{2}}{\partial y^{2}}\right)+\left(\frac{\partial^{2}}{\partial z^{2}}\right)\right]
\end{aligned}
$$

is the dissipative term, $\delta t / \tau$ is the scale resolution identified here with $d t / \tau$ by substitution principle [12-14], $D_{F}$ is the fractal dimension of the movement curves, $\lambda$ is the reference length scale, $\tau$ is the reference time scale, and $\lambda^{2} / \tau \equiv D$ is the Nottale coefficient specific to fractal-nonfractal transition [12-14]. In the case of fractal dimension $D_{F}$, we can use any definition (the Hausdorff-Besicovitch fractal dimension, the Kolmogorov fractal dimension, etc. [11]), but once such definition is accepted, it has to be constant over the entire complex system dynamics analysis.

Applying the fractal operator (1) to the complex velocity (2) and accepting a generalized inertial principle (a generalization of Nottale's principle of scale covariance [12-14]), we obtain the geodesics in the form of the Navier-Stokes-type equations:

$$
\frac{\widehat{d} \widehat{\mathbf{V}}}{d t}=\frac{\partial \widehat{\mathbf{V}}}{\partial t}+(\widehat{\mathbf{V}} \nabla) \widehat{\mathbf{V}}-i \frac{\lambda^{2}}{\tau}\left(\frac{d t}{\tau}\right)^{\left(2 / D_{F}\right)-1} \Delta \widehat{\mathbf{V}}=0 .
$$

Equation (4) shows that at any point of a fractal path, local acceleration, $\partial_{t} \widehat{\mathbf{V}}$, convection, $(\widehat{\mathbf{V}} \nabla) \widehat{\mathbf{V}}$, and dissipation, $\left(\lambda^{2} / \tau\right)(d t / \tau)^{\left(2 / D_{F}\right)-1} \Delta \widehat{\mathbf{V}}$, is in equilibrium. According to [26], the complex system can be assimilated to a "rheological" fractal fluid. The "rheology" of the fractal fluid gives hysteretic 
properties to the complex system (the complex system has a hysteresis cycle, memory, etc.).

Since the movement of the complex system's entities lacks interaction, we practically make use of self-convection and self-dissipation type mechanisms.

\section{Fractal Hydrodynamics Model}

For irrotational motion

$$
\begin{aligned}
& \nabla \times \widehat{\mathbf{V}}=0, \\
& \nabla \times \mathbf{V}_{D}=0, \\
& \nabla \times \mathbf{V}_{F}=0
\end{aligned}
$$

we can choose $\widehat{\mathbf{V}}$ of the form

$$
\widehat{\mathbf{V}}=-2 i \frac{\lambda^{2}}{\tau}\left(\frac{d t}{\tau}\right)^{\left(2 / D_{F}\right)-1} \nabla \ln \psi
$$

or explicitly, with $\psi=\sqrt{\rho} \exp (i S)$,

$$
\begin{gathered}
\widehat{\mathbf{V}}=2 \frac{\lambda^{2}}{\tau}\left(\frac{d t}{\tau}\right)^{\left(2 / D_{F}\right)-1} \nabla S-i \frac{\lambda^{2}}{\tau}\left(\frac{d t}{\tau}\right)^{\left(2 / D_{F}\right)-1} \nabla \ln \rho, \\
\mathbf{V}_{D}=2 \frac{\lambda^{2}}{\tau}\left(\frac{d t}{\tau}\right)^{\left(2 / D_{F}\right)-1} \nabla S, \\
\mathbf{V}_{F}=\frac{\lambda^{2}}{\tau}\left(\frac{d t}{\tau}\right)^{\left(2 / D_{F}\right)-1} \nabla \ln \rho,
\end{gathered}
$$

where $\rho$ is an amplitude and $S$ a phase. The function $\psi$ by means of ln defines the velocity scalar potential

$$
\Phi=2 \frac{\lambda^{2}}{\tau}\left(\frac{d t}{\tau}\right)^{\left(2 / D_{F}\right)-1} S-i \frac{\lambda^{2}}{\tau}\left(\frac{d t}{\tau}\right)^{\left(2 / D_{F}\right)-1} \ln \rho .
$$

By substituting $((7 a)-(7 c))$ in (4) and separating the real and imaginary parts, up to an arbitrary phase factor which may be at zero by a suitable choice of the phase $\psi$, we obtain

$$
\begin{gathered}
m_{0}\left[\frac{\partial \mathbf{V}_{D}}{\partial t}+\left(\mathbf{V}_{D} \cdot \nabla\right) \mathbf{V}_{D}\right]=-\nabla Q \\
\frac{\partial \rho}{\partial t}+\nabla\left(\rho \mathbf{V}_{D}\right)=0
\end{gathered}
$$

with $Q$ being the fractal potential

$$
\begin{aligned}
Q & =-2 m_{e} \frac{\lambda^{4}}{\tau^{2}}\left(\frac{d t}{\tau}\right)^{\left(4 / D_{F}\right)-2} \frac{\Delta \sqrt{\rho}}{\rho} \\
& =-\frac{m_{0} \mathbf{V}_{F}^{2}}{2}-m_{0} \frac{\lambda^{2}}{\tau}\left(\frac{d t}{\tau}\right)^{\left(2 / D_{F}\right)-1} \nabla \mathbf{V}_{F}
\end{aligned}
$$

and $m_{0}$ the rest mass of the complex system's entity. The first equation (9) is the momentum conservation law, the second equation (10) is the density conservation law, and they define together the fractal hydrodynamics (FH) model. We note that, for Peano-type movements in fractal dimension $D_{F}=2$ and supposing that fractal potential is assimilated with the pressure, the fractal hydrodynamic model becomes the standard hydrodynamic (for details see [19-23]).

The fractal potential (11) comes from the nondifferentiability and must be considered as a kinetic term and not as a potential one. Moreover, the fractal potential (11) can generate a viscosity stress tensor type $[22,23]$

$$
\begin{aligned}
\widehat{\sigma}_{i l} & =m_{0} \frac{\lambda^{4}}{\tau^{2}}\left(\frac{d t}{\tau}\right)^{\left(4 / D_{F}\right)-2}\left[\nabla_{i} \rho \nabla_{l} \rho-\left(\frac{\nabla_{i} \rho \nabla_{l} \rho}{\rho}\right)\right] \\
& =\eta\left(\frac{\partial V_{F i}}{\partial x_{l}}+\frac{\partial V_{F l}}{\partial x_{i}}\right), \quad \eta=\frac{1}{2} m_{0} \rho \frac{\lambda^{2}}{\tau}\left(\frac{d t}{\tau}\right)^{\left(2 / D_{F}\right)-1}
\end{aligned}
$$

of which divergence is equal to the usual force density associated with $Q$

$$
\nabla_{i} \widehat{\sigma}_{i l}=-\rho \nabla_{l} Q
$$

\section{Barotropic-Type Behaviours of the Complex Systems via Nondifferentiability}

For a barotropic-type behaviour of the complex system [24],

$$
\widehat{\sigma}_{i l}=\sigma \delta_{i l}=\rho c^{2} \delta_{i l}
$$

with $c$ being the critical velocity (for details see [24]); the FH equations become

$$
\begin{gathered}
{\left[\frac{\partial \mathbf{V}_{D}}{\partial t}+\left(\mathbf{V}_{D} \cdot \nabla\right) \mathbf{V}_{D}\right]=-\frac{c^{2}}{\rho} \nabla \rho} \\
\frac{\partial \rho}{\partial t}+\nabla\left(\rho \mathbf{V}_{D}\right)=0
\end{gathered}
$$

In the following, using (15a) and (15b) in a plane symmetry, we analyze the complex fluid dynamics. The presence of an external field is specified by adequate initial and boundary conditions (e.g., spatial-temporal Gaussian). In this situation, let us introduce the normalized coordinates

$$
\begin{gathered}
\omega t=\tau_{0}, \\
k x=\xi, \\
k y=\eta, \\
\frac{V_{x} k}{\omega}=V_{\xi}, \\
\frac{V_{y} k}{\omega}=V_{\eta}, \\
\frac{\rho}{\rho_{0}}=N,
\end{gathered}
$$

where $\omega, k$, and $\rho_{0}$ are critical parameters of the complex fluid (in $[19,25-27]$ the complex fluid is identified with a laser 
produced plasma; in such a context $\omega$ is the plasma pulsation, $\rho_{0}$ is the density of plasma at thermodynamic equilibrium, $k$ is the inverse of the Debye length, and $c$ is the ion acoustic speed).

Then (15a) and (15b) become

$$
\begin{gathered}
\frac{\partial}{\partial \tau_{0}}\left(N V_{\xi}\right)+\frac{\partial}{\partial \xi}\left(N V_{\xi}^{2}\right)+\frac{\partial}{\partial \eta}\left(N V_{\xi} V_{\eta}\right)=-N^{-1} \frac{\partial N}{\partial \xi} \\
\frac{\partial}{\partial \tau_{0}}\left(N V_{\eta}\right)+\frac{\partial}{\partial \xi}\left(N V_{\xi} V_{\eta}\right)+\frac{\partial}{\partial \eta}\left(N V_{\eta}^{2}\right)=-N^{-1} \frac{\partial N}{\partial \eta} \\
\frac{\partial N}{\partial \tau_{0}}+\frac{\partial}{\partial \xi}\left(N V_{\xi}\right)+\frac{\partial}{\partial \eta}\left(N V_{\eta}\right)=0
\end{gathered}
$$

For the numerical integration we will impose the initial conditions

$$
\begin{gathered}
V_{\xi}(0, \xi, \eta)=0, \\
V_{\eta}(0, \xi, \eta)=0, \\
N(0, \xi, \eta)=\frac{1}{5}, \\
1 \leq \xi \leq 2, \\
0 \leq \eta \leq 1
\end{gathered}
$$

as well as the boundary conditions

$$
\begin{array}{cl}
V_{\xi}\left(\tau_{0}, 1, \eta\right)=V_{\xi}\left(\tau_{0}, 2, \eta\right)=0, & (19 \mathrm{a}) \\
V_{\eta}\left(\tau_{0}, 1, \eta\right)=V_{\eta}\left(\tau_{0}, 2, \eta\right)=0, & (19 \mathrm{~b}) \\
V_{\xi}\left(\tau_{0}, \xi, 0\right)=V_{\xi}\left(\tau_{0}, \xi, 1\right)=0, & (19 \mathrm{c}) \\
V_{\eta}\left(\tau_{0}, \xi, 0\right)=V_{\eta}\left(\tau_{0}, \xi, 1\right)=0, \\
N\left(\tau_{0}, 1, \eta\right)=N\left(\tau_{0}, 2, \eta\right)=\frac{1}{5}, \\
N\left(\tau_{0}, \xi, 0\right)=\frac{1}{10} \exp \left[-\left(\frac{\tau_{0}-1 / 5}{1 / 5}\right)^{2}\right] \exp \left[-\left(\frac{\xi-3 / 2}{1 / 5}\right)^{2}\right], \\
N\left(\tau_{0}, \xi, 1\right)=\frac{1}{5} .
\end{array}
$$

By using the finite differences method [28], the system $((17 a)-(17 c))$ with the initial conditions $((18 a)-(18 e))$ and the boundary ones $((19 a)-(19 g))$ was numerically resolved.

In Figures 1(a), 1(b), and 1(c), three-dimensional dependences of the normalized density $N$, normalized velocities, $V_{\xi}$ and $V_{\eta}$, on the normalized coordinates, $\xi$ and $\eta$, are given for the normalized time $\tau_{0}=0.54$. Also in Figures $1(\mathrm{~d})$, $1(\mathrm{e})$, and $1(\mathrm{f})$ the two-dimensional contours of the normalized density $N$, and normalized velocities, $V_{\xi}$ and $V_{\eta}$, are given for the same normalized time. The followings result in (i) the generation of two complex fluid structures; (ii) the symmetry of the normalized velocity, $V_{\xi}$, with respect to symmetry axis of the spatial-temporal Gaussian; and (iii) vertices at the complex fluid periphery for the normalized velocity field, $V_{\eta}$. These results are in agreement with the experimental data on the behaviours of a laser produced aluminium plasma $[19,26,27,29-33]$.

Starting from these numerical solutions we can build the normalized current density

$$
j\left(\xi, \eta, \tau_{0}\right)=N\left(\xi, \eta, \tau_{0}\right)\left[V_{\xi}^{2}\left(\xi, \eta, \tau_{0}\right)+V_{\xi}^{2}\left(\xi, \eta, \tau_{0}\right)\right]^{1 / 2}
$$

See Figures 2(a)-2(c).

It results in the splitting of the complex fluid into two structures. By assimilating the complex fluid with a laser produced aluminium plasma, the previous theoretical results are in agreement with the experimental data $[32,33]$.

\section{Self-Similarity Behaviour of the Complex Systems via Nondifferentiability}

Neglecting both convection $\widehat{\mathbf{V}} \cdot \nabla \widehat{\mathbf{V}}$ and dispersion $\left(\lambda^{3} / \tau\right)$ $(d t / \tau)^{\left(3 / D_{F}\right)-1} \nabla^{3} \widehat{\mathbf{V}},(4)$ takes the form

$$
\frac{d \widehat{\mathbf{V}}}{d t}=\frac{\partial \widehat{\mathbf{V}}}{\partial t}-i \frac{\lambda^{2}}{\tau}\left(\frac{d t}{\tau}\right)^{\left(2 / D_{F}\right)-1} \Delta \widehat{\mathbf{V}}=0
$$

or, by separating the resolution scales,

$$
\frac{\partial \mathbf{V}_{D}}{\partial t}-\frac{\lambda^{2}}{\tau}\left(\frac{d t}{\tau}\right)^{\left(2 / D_{F}\right)-1} \Delta \mathbf{V}_{F}=0
$$

for the differentiable scale and

$$
\frac{\partial \mathbf{V}_{F}}{\partial t}-\frac{\lambda^{2}}{\tau}\left(\frac{d t}{\tau}\right)^{\left(2 / D_{F}\right)-1} \Delta \mathbf{V}_{D}=0
$$

for fractal scale. The velocity fields are totally separated, first applying (22) and (23) to the $\Delta$ operator, that is,

$$
\begin{aligned}
& \frac{\partial}{\partial t}\left(\Delta \mathbf{V}_{D}\right)-\frac{\lambda^{2}}{\tau}\left(\frac{d t}{\tau}\right)^{\left(2 / D_{F}\right)-1} \Delta^{2} \mathbf{V}_{F}=0, \\
& \frac{\partial}{\partial t}\left(\Delta \mathbf{V}_{F}\right)+\frac{\lambda^{2}}{\tau}\left(\frac{d t}{\tau}\right)^{\left(2 / D_{F}\right)-1} \Delta^{2} \mathbf{V}_{D}=0,
\end{aligned}
$$

then, substituting in the transformed equations the dissipative terms by using (22) and (23). It results in the Kirchhofftype equations

$$
\left(\frac{\partial^{2}}{\partial t^{2}}+\frac{\lambda^{4}}{\tau^{2}}\left(\frac{d t}{\tau}\right)^{\left(4 / D_{F}\right)-2} \Delta^{2}\right)\left(\begin{array}{l}
\mathbf{V}_{D} \\
\mathbf{V}_{F}
\end{array}\right)=\left(\begin{array}{l}
0 \\
0
\end{array}\right)
$$




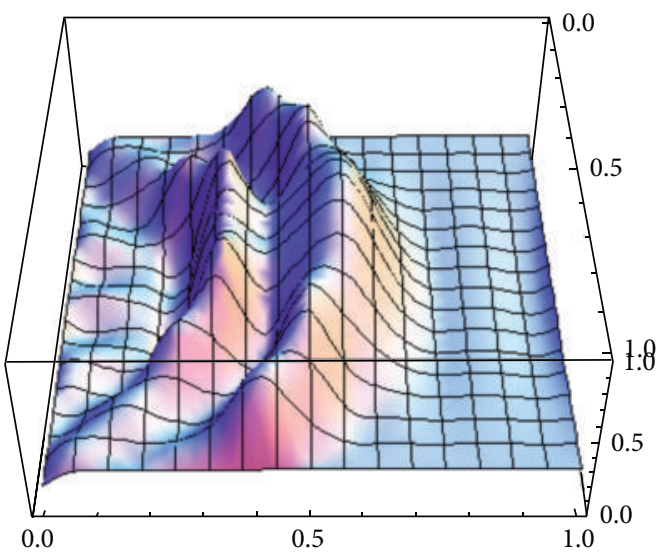

(a)

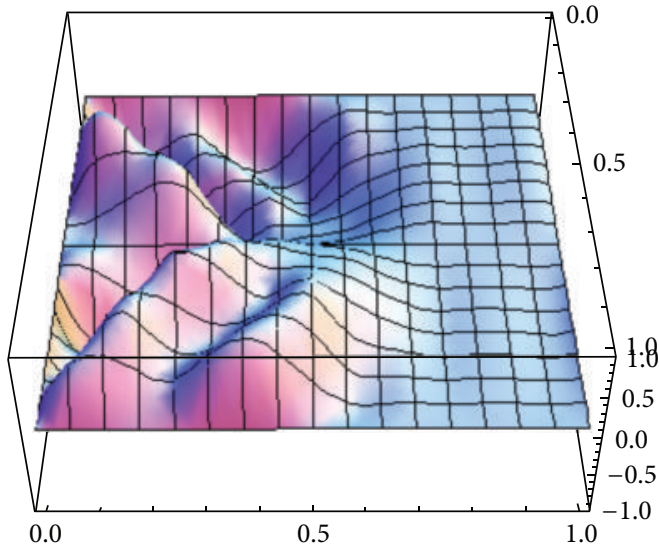

(b)
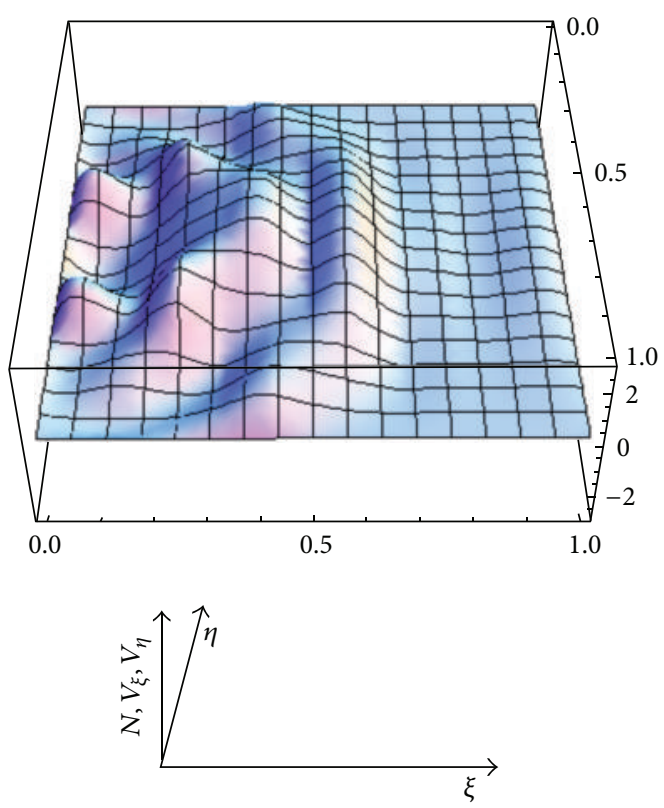

(c)

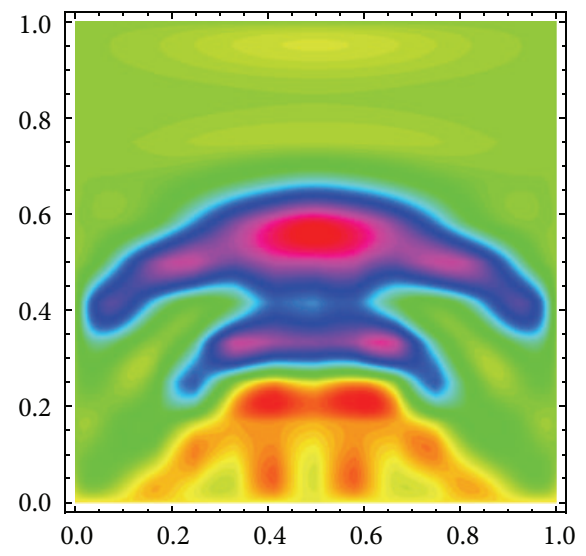

(d)

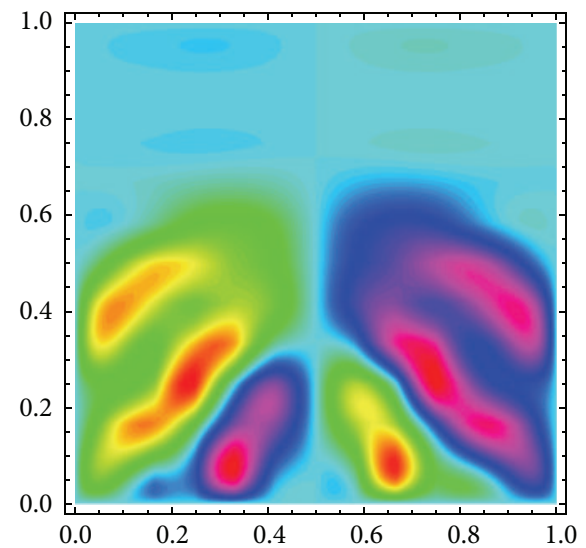

(e)
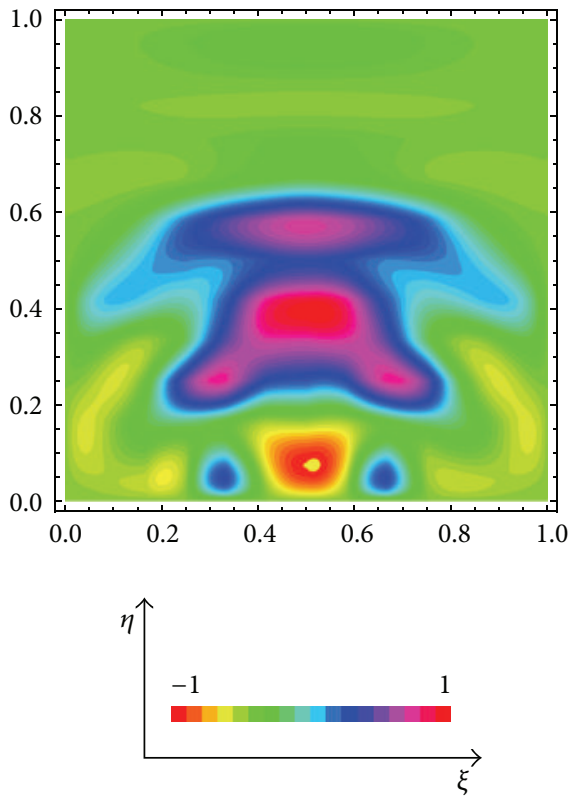

(f)

FIGURE 1: Three-dimensional dependences of the normalized density $N$ and normalized velocities, $V_{\xi}$ and $V_{\eta}$, on the normalized coordinates, $\xi$ and $\eta$, for the normalized time $\tau_{0}=0.54$ ((a), (b), and (c)); two-dimensional contour of the normalized density $N$ and normalized velocities, $V_{\xi}$ and $V_{\eta}$, for the same normalized time ((d), (e), and (f)). 


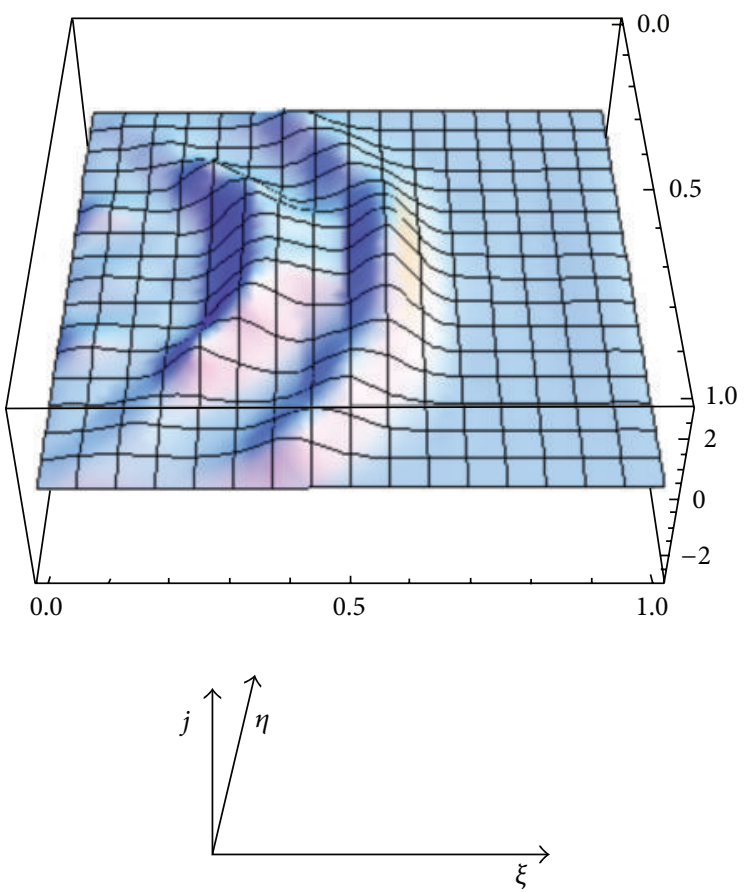

(a)
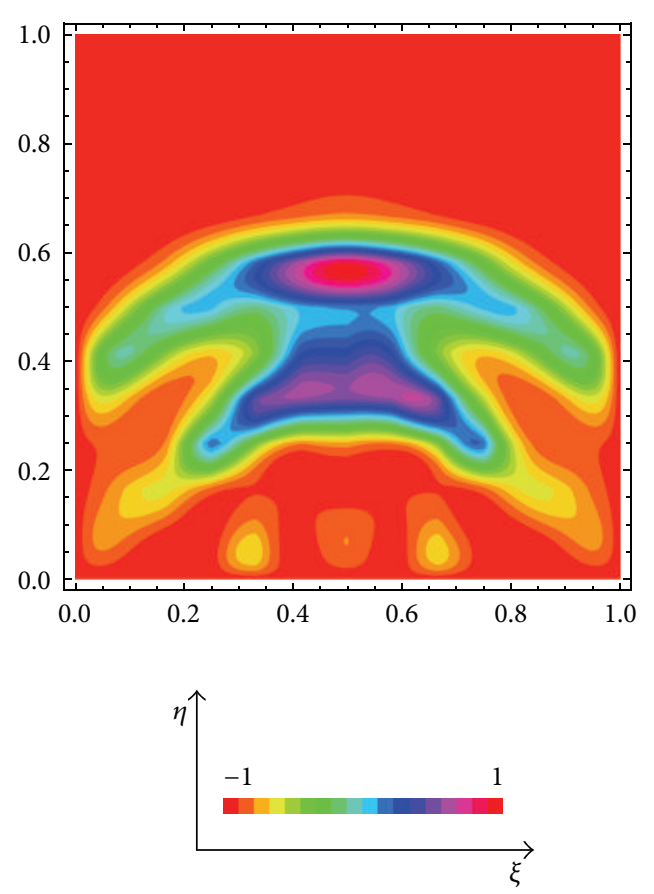

(b)

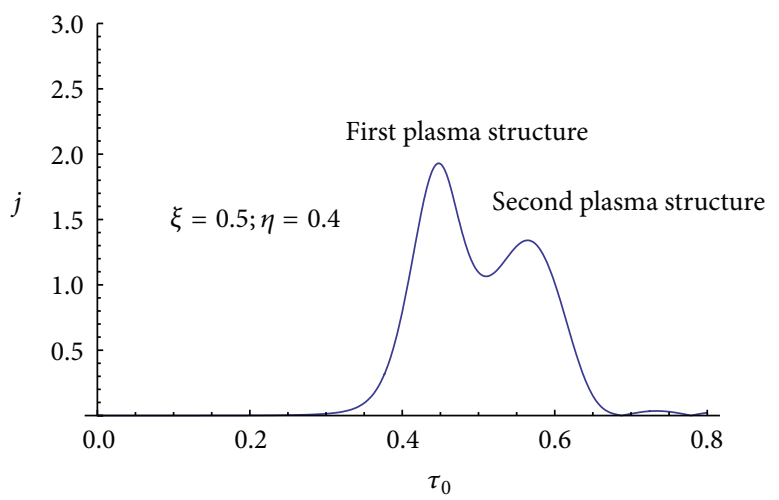

(c)

FiguRE 2: Three-dimensional dependences of the normalized density current $j$, on the normalized coordinates, $\xi$ and $\eta$, for the normalized time $\tau_{0}=0.54$ (a); two-dimensional contour of the normalized current density $j$ for the same normalized time (b); time evolution of the normalized density current for $\xi=0.5$ and $\eta=0.4$ (c).

For one-dimensional case, the previous equations with the substitutions

$$
\begin{gathered}
\frac{x}{L}=\bar{\xi} \\
\frac{t}{T}=\bar{\tau} \\
\frac{L^{4}}{T^{2}}=\frac{\lambda^{4}}{\tau^{2}}\left(\frac{d t}{\tau}\right)^{\left(4 / D_{F}\right)-2}, \\
\left(\mathbf{V}_{D} ; \mathbf{V}_{F}\right) \equiv \bar{K}_{i}(\bar{\xi}, \bar{\tau}), \quad i=1,2
\end{gathered}
$$

take the unitary form

$$
L^{4} \frac{\partial^{4} \bar{K}_{i}}{\partial \bar{\xi}^{4}}+T^{2} \frac{\partial^{2} \bar{K}_{i}}{\partial \bar{\tau}^{2}}=0
$$

On (27), we impose for "clamping” conditions at $\bar{\xi}=1$

$$
\frac{\partial^{2} \bar{K}_{i}(1, \bar{\tau})}{\partial \bar{\xi}^{2}}=0,
$$




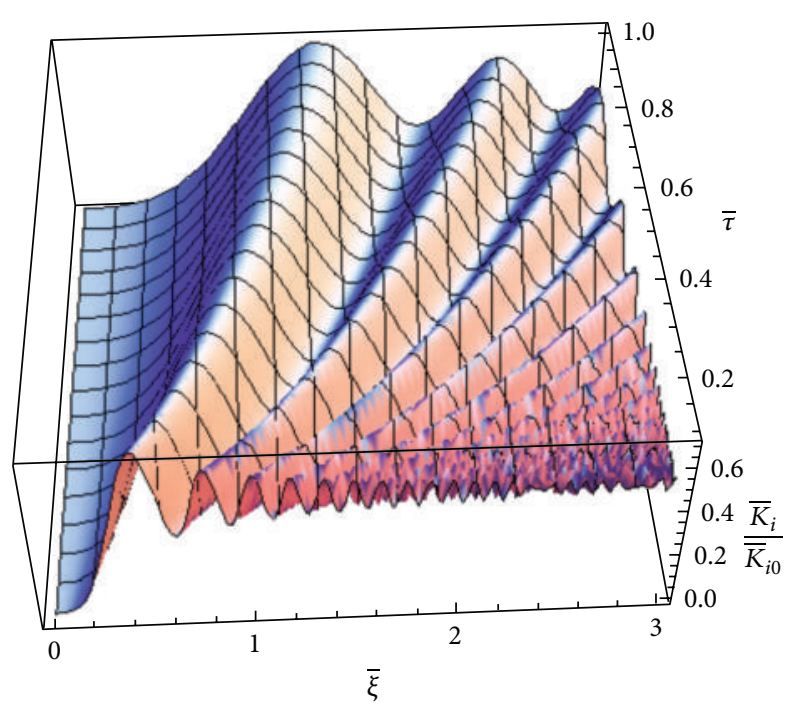

FIgURE 3: Numerical solution of the Kirchhoff equation (27) with "clamped-free" unitary conditions, for a uniform initial $\bar{K}_{i 0} \cdot \bar{K}_{i}(0, \tau)$ relaxes to zero within the first few time steps.

$$
\frac{\partial^{3} \bar{K}_{i}(1, \bar{\tau})}{\partial \bar{\xi}^{3}}=0
$$

and for boundary conditions at $\bar{\xi}=0$

$$
\begin{gathered}
\bar{K}_{i}(0, \bar{\tau})=0, \\
\frac{\partial \bar{K}_{i}(0, \bar{\tau})}{\partial \bar{\xi}}=0 .
\end{gathered}
$$

These four boundary conditions in $\bar{\xi}$ associated with the two initial conditions

$$
\begin{gathered}
\bar{K}_{i}(\bar{\xi}, 0)=\bar{K}_{i 0} \\
\frac{\partial \bar{K}_{i}(\bar{\xi}, 0)}{\partial \bar{\tau}}=0
\end{gathered}
$$

imply a unique solution $\bar{K}_{i}(\bar{\xi}, \bar{\tau})$ to (27); see Figure 3 .

Owing to the scaling $x \sim L \sqrt{t / T}$, we seek a solution of (27) in the form

$$
\bar{K}_{i}(\bar{\xi}, \bar{\tau})=\bar{K}_{i 0} u_{i}(\bar{\eta})
$$

where the self-similarity variable is

$$
\bar{\eta}=\left(\frac{\bar{\xi}}{\sqrt{\bar{\tau}}}\right)=\frac{(x / L)}{\sqrt{t / T}}=\frac{x}{\sqrt{\gamma t}} .
$$

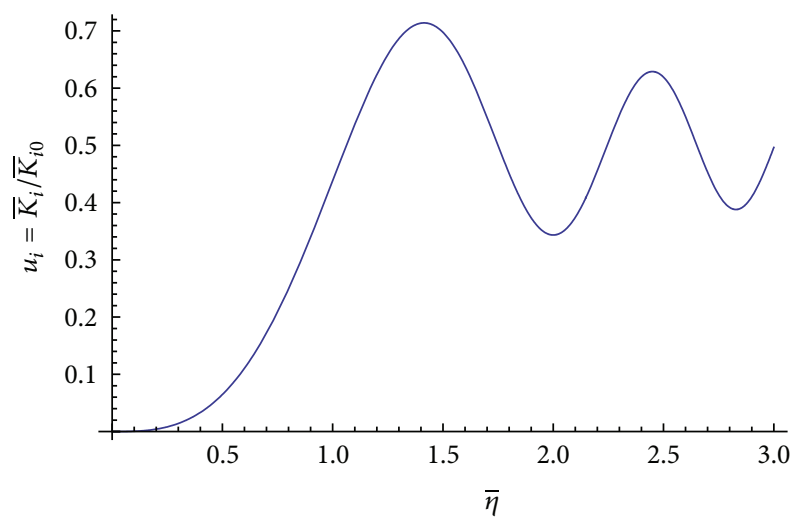

FIgURE 4: The self-similar solution (36) is a function of $\bar{\eta}$. At later times, $\bar{\tau} \sim 1$, reflections are generated from the "clamped" end $\bar{\xi}=1$.

The boundary conditions for $u(\bar{\eta})$ are derived from those for $\bar{K}_{i}$ :

$$
\begin{gathered}
u_{i}(0)=0, \\
u_{i}^{\prime}(0)=0, \\
u_{i}(+\infty) \longrightarrow 1 .
\end{gathered}
$$

Substituting this self-similar form of $\bar{K}_{i}(\bar{\xi}, \bar{\tau})$ into (27) yields the following equation for the self-similar solution $u_{i}(\bar{\eta})$ :

$$
4 \frac{d^{4} u_{i}(\bar{\eta})}{d \bar{\eta}^{4}}+\bar{\eta}^{2} \frac{d^{2} u_{i}(\bar{\eta})}{d \bar{\eta}^{2}}+3 \bar{\eta} \frac{d u_{i}(\bar{\eta})}{d \bar{\eta}}=0
$$

Imposing that $u_{i}(\bar{\eta})$ matches the initial condition for large $\bar{\eta}$ implies that

$$
\frac{d^{2} u_{i}(0)}{d \bar{\eta}^{2}}=0
$$

as shown with the help of an integral of motion. This last condition, combined with the previous ones, yields a unique self-similar solution to (34):

$$
2 \bar{K}_{i}(\bar{\xi}, \bar{\tau})=2 \bar{K}_{i 0}\left(\frac{\bar{\eta}}{\sqrt{2 \pi}}\right)
$$

where we have introduced the Fresnel sine integral, $S(x)=$ $\int_{0}^{x} \sin \left(\pi y^{2} / 2\right) d y$, also arising in diffraction theory.

Equation (36) describes a self-similar solution. This reflects the dispersive nature of (27), see Figure 4.

In these conditions the phase $S$ associated with the velocity scalar potential is presented in Figure 5.

The states density associated with the same velocity scalar potential has the same behaviour as the one presented in Figure 5. It results in a self-similarity behaviour.

The theoretical oscillations (obtained by means of selfelimination of the dissipative terms between differentiable 


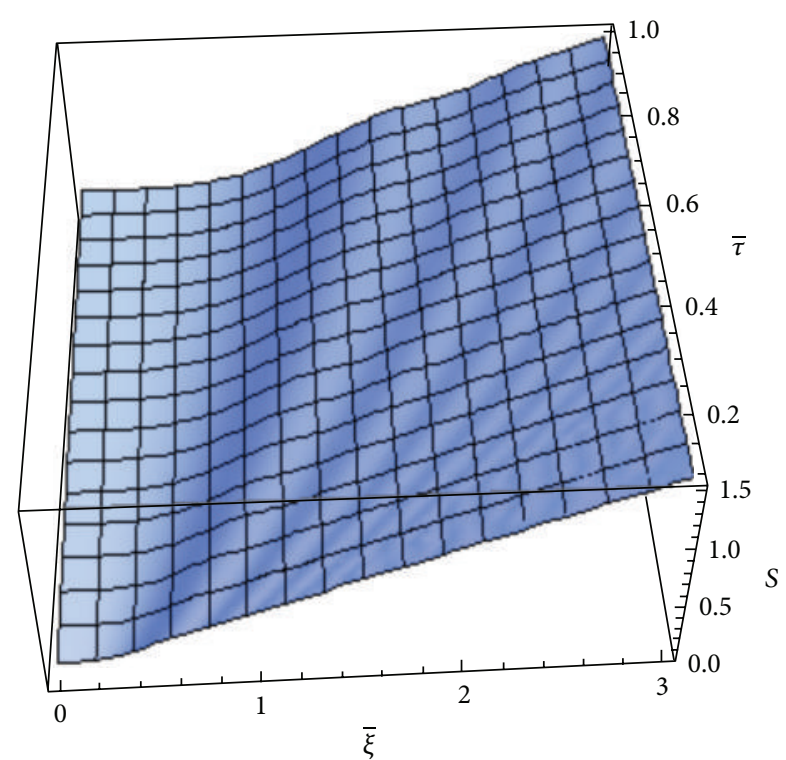

FIGURE 5: The phase dependence versus normalized space-time coordinates.

and fractal scales) can explain the experimental ionic oscillations of the current density that take place in a laser produced aluminium plasma $[19,26,27,29]$. In Figure 6 we give by comparison the experimental curve (red curve, which reflects the ionic oscillations of the current density with time that take place in a laser produced aluminium plasma at a laser power of $40 \mathrm{~mJ} /$ pulse) and the theoretical curve (blue curve). We note that in Figure 6 we used the normalized coordinates.

It results in a good agreement (correlation factor 0.81) between theoretical model and the experimental data.

\section{Chaoticity through Turbulence and Stochasticization via Nondifferentiability}

Through the fractal velocities field, $\mathbf{V}_{F}$, the specific fractal potential $Q$ is a measure of nondifferentiability of the complex system particle trajectories, that is, of their chaoticity.

Since the position vector of the particle is assimilated with a stochastic process of Wiener type (for details see [11-13], $\psi$ is not only the scalar potential of a complex speed (through $\ln \psi$ ) in the frame of fractal hydrodynamics but also density of probability (through $|\psi|^{2}$ ) in the frame of a Schrödinger-type theory.

It results in the equivalence between the formalism of the fractal hydrodynamics and the one of the Schrödinger-type equation. Moreover, the chaoticity, either through turbulence in the fractal hydrodynamics approach or through stochasticization in the Schrödinger-type approach, is generated only by the nondifferentiability of the movement trajectories in a fractal space. In this way the nondifferentiability becomes a "control parameter" of the complex system dynamics.

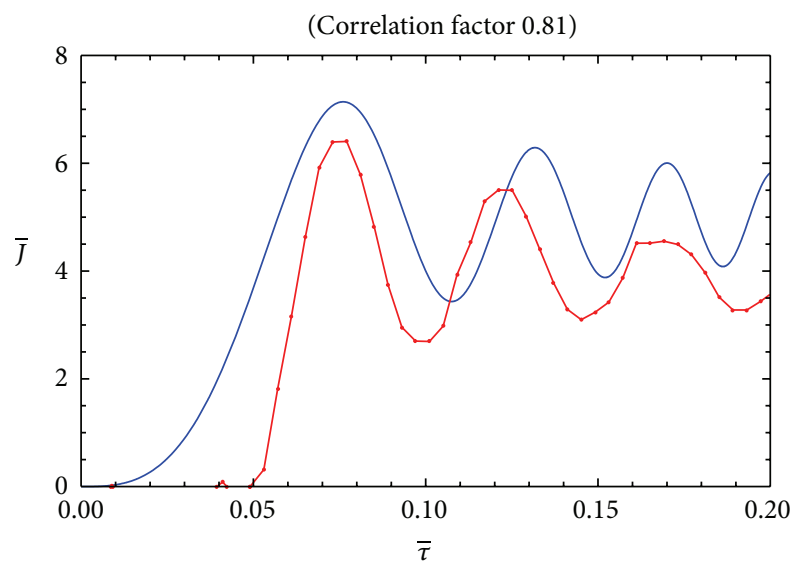

Figure 6: The theoretical (blue) and experimental (red) curves of the ionic oscillations of the normalized current density, $\bar{J}$, with normalized time, $\bar{\tau}$, in a laser produced aluminium plasma at a laser power of $40 \mathrm{~mJ} /$ pulse.

\subsection{Full and Fractional Speed Scalar Potential Revivals in the Infinite Square Well: Various Criteria of Evolution to Chaos}

6.1.1. Infinite Square Well System. Let us consider that the external perturbation applied to complex system simulates, in our opinion, one-dimension square well system. After solving the time-dependent Schrödinger-type equation according to the method described in [23] we obtain the discrete eigenvalues

$$
E_{n}=2 m_{0} \mathfrak{D}^{2}\left(\frac{n \pi}{a}\right)^{2}, \quad \mathfrak{D}=\frac{\lambda^{2}}{\tau}\left(\frac{d t}{\tau}\right)^{\left(2 / D_{F}\right)-1}
$$

and the eigenfunctions

$$
\phi_{n}= \begin{cases}\left(\frac{2}{a}\right)^{1 / 2} \sin \left(\frac{n \pi x}{a}\right), & n \text { even }|x| \leq \frac{a}{2}, \\ \left(\frac{2}{a}\right)^{1 / 2} \cos \left(\frac{n \pi x}{a}\right), & n \text { odd }|x| \leq \frac{a}{2}\end{cases}
$$

where $a$ is the well's width and $m_{0}$ is the rest mass of the fractal fluid particle.

6.1.2. Time Scales. Some time scales of a speed potential evolution are contained in the coefficients of the Taylor series of the quantized energy levels $E_{n}$ around the main energy $E_{\bar{n}}$ (see method from [34])

$$
E_{n}=E_{\bar{n}}+4 \pi m_{0} \mathfrak{D}\left[\frac{n-\bar{n}}{T_{\alpha}}+\frac{(n-\bar{n})^{2}}{T_{\beta}}+\cdots\right],
$$

where often the zero of energy is shifted to remove the $E_{\bar{n}}$ term. Regrouping the infinite square well energies (17a)-(17c) in this form gives

$$
E_{n}=E_{1} n^{2}=E_{1} \bar{n}^{2}+2 E_{1} \bar{n}(n-\bar{n})+E_{1}(n-\bar{n})^{2} .
$$


And comparing (39) and (40) we relate

$$
\begin{aligned}
T_{\alpha} & =\frac{2 \pi m_{0} \mathfrak{D}}{\bar{n} E_{1}}, \\
T_{\beta} & =\frac{4 \pi m_{0} \mathfrak{D}}{E_{1}} .
\end{aligned}
$$

We note that the time scale $T_{\beta}$ does not depend on the mean energy level $\bar{n}$. This will provide us with a "universal" time scale for describing speed potential evolution that does not depend on the particle average energy.

6.1.3. Time Evolution. We write the particle's time $t=0$ speed scalar potential in the infinite square well as

$$
\psi(x, t=0)=\psi_{i}(x) .
$$

We expand this speed scalar potential using the energy eigenstate basis

$$
\psi_{i}(x)=\sum_{n=1}^{\infty} c_{n} \phi_{n}(x)
$$

with

$$
c_{n}=\int_{-\infty}^{+\infty} \phi_{n}(x) \psi_{i}(x) d x
$$

Using the time scale $T_{\beta}$, the time evolution in the energy eigenstate basis was found from Schrödinger-type equation to be

$$
\psi(x, t)=\sum_{n} \exp \left[-2 \pi i\left(\frac{t}{T_{\beta}}\right) n^{2}\right] c_{n} \phi_{n}(x) .
$$

6.1.4. Simulation of the Evolution to Chaos Criteria. Now, the full and fractional revivals formalism may be applied. Full and fractional revivals of a speed scalar potential in the infinite square well occur when a speed scalar potential evolves in time to a state that can be described as a collection of spatially distributed subspeed scalar potentials that each closely reproduces the initial speed scalar potential shape; see for details [34]. Therefore, the full and fractional revivals of a speed scalar potential in the infinite square well impliy either

$$
\psi\left(x, t=t_{0}+2^{k} T_{\beta}\right)=\psi\left(x, t=t_{0}\right)
$$

or

$$
\psi\left(x, t_{0}+\frac{p}{q} T_{\beta}\right)=\psi\left(x, t=t_{0}\right)
$$

for any time $t_{0}$ and $k, p$, and $q$ integers. In any of the situations above, either for $t=T_{F}=2^{k} T_{\beta}$ or for $t=T_{\mathrm{SH}}=(p / q) T_{\beta}$, we can introduce Reynolds-type criterions

$$
\begin{gathered}
\operatorname{Re}_{F}=\frac{V_{F} L_{F}}{v_{F}}=2^{k}, \\
\operatorname{Re}_{\mathrm{SH}}=\frac{V_{\mathrm{SH}} L_{\mathrm{SH}}}{v_{\mathrm{SH}}}=\frac{p}{q},
\end{gathered}
$$

where

$$
\begin{gathered}
E_{1} \equiv E_{F / S H}=\frac{1}{2} m_{0} V_{F / S H}^{2}, \\
L_{F / S H}=V_{F / S H} T_{F / S H}, \\
\nu_{F / S H}=8 \pi \frac{\lambda^{2}}{\tau}\left(\frac{d t}{\tau}\right)^{\left(2 / D_{F}\right)-1}
\end{gathered}
$$

have the usual signification from fluid mechanics [24]. Up to the values $\operatorname{Re}_{F / S H}^{c}$ the fractal fluids become turbulent. Then through $T_{F} / T_{\beta}=2^{k}$ and $\operatorname{Re}_{F}=V_{F} L_{F} / \nu_{F}=2^{k}$ it is formally simulated through the criterion of evolution to chaos via Feigenbaum scenario (cascade of period doubling bifurcations), while through $T_{\beta} / T_{\mathrm{SH}}=\omega_{\mathrm{SH}} / \omega_{\beta}=q / p$ with $p>q$ and $\mathrm{Re}_{\mathrm{SH}}=V_{\mathrm{SH}} L_{\mathrm{SH}} / \nu_{\mathrm{SH}}=p / q$ the criterion of evolution to chaos via a cascade of subharmonic bifurcations.

We admit that in any of these two situations mentioned above the fractal velocity (7c) is null, since $\rho=|\psi|^{2}=$ const; meanwhile the differential velocity $(7 \mathrm{~b})$ is not zero, since the phase $S$ is not constant, with the increase of the systems phase incoherence being associated with the increase in turbulence of fractal fluid.

We note that in the standard model (Landau's scenario $[24,35])$ the Fourier spectrum is always discrete and cannot approximate a continuum spectrum than that in case of a large number of frequencies that will generate an unlimited number of spectral components as a result of their beats which appear thanks to the presence of nonlinearities in the system. Yet, considering standard model, the flow can never be truly chaotic, because, in case of multiple periodic functions, correlations tend to be not null but having an oscillating character. Therefore, Landau's scenario can describe transition towards chaotic behaviour only in a system with an infinite number of degrees of freedom, such as a fluid. In our case, because the Reynolds numbers (48) present scale dependencies (49c), when $d t \rightarrow 0$ for $D_{F} \leq 2$, the fractals physical values that describe the dynamics of the system are no longer defined. So, in this approximation, a simulation of a system with an infinite number of degrees of freedom is used.

We note that the results from the present paragraph permit the chaoticity and self-structuring control by means of nondifferentiability. Thus the experimental results on the formation, dynamics, and evolution towards chaos of complex space charge structures that emerge in front of a positively biased electrode immersed in a quiescent plasma from [20] are in agreement with our previous theoretical results.

\section{Conclusions}

In the present paper, we propose a new topic in the control of complex systems dynamics using the nondifferentiability of complex system movement curves. This topic was developed through the scale relativity approach. Considering the dynamics of complex system entities that take place on fractal curves, we show that the control of different behaviours of these systems implies nondifferentiability. The 
main conclusions of the present paper are as follows: (i) the geodesics equations in the form of a Navier-Stokes-type equation are obtained. It results in the rheologic properties of the complex system which implies memory and so forth; (ii) considering that the fractal fluid flows are irrotational, fractal hydrodynamics equations are obtained. These equations are formed from the density and momentum conservations laws, wih the presence of nondifferentiability being induced by the fractal potential; (iii) a barotropic-type behaviour of the complex system is numerically simulated using FH equations. In this way we highlight the self-multiplication mechanism of the substructures that constitute the complex system; (iv) the implications of self-similarity in the dynamics of the complex system are presented; (v) the chaoticity through turbulence and stochaticization via nondifferentiability are obtained; (vi) these previous behaviours can simulate the standard properties of the complex system.

In this way, standard properties of complex systems such as emergence, self-organisation, and adaptability are controlled through nondifferentiability of motion curves of the sub-systems that compose the complex system. We note that general aspects of the dynamics control of the complex system are described in [36], while the concrete cases of this control are presented in [37-39].

\section{Appendices}

\section{A. Consequences of Nondifferentiability}

The nondifferentiability implies the following [12-14, 19-22].

(i) A continuous and a nondifferentiable curve (or almost nowhere differentiable) is explicitly scale dependent. This means that its length tends to infinity, when the scale interval tends to zero. Therefore, a continuous and nondifferentiable space is fractal, in the general meaning given by Mandelbrot to this concept [11].

(ii) Physical quantities will be expressed through fractal functions, namely, through functions that are dependent both on spatial and temporal coordinates and on resolution scale. The invariance of the physical quantities in relation with the resolution scale generates special types of transformations, called resolution scale transformations. Particularly, the differentiality of the generalized spatial coordinates, $\mathbf{X}$, takes the form

$$
d \mathbf{X}=d \mathbf{x}+d \boldsymbol{\xi}
$$

where $d \mathbf{x}$ is the classical differential element and $d \boldsymbol{\xi}$ is a differential fractal one.

(iii) There is infinity of fractal curves (geodesics) relating to any couple of points (or starting from any point) and applied for any scale. The phenomenon can be easily understood at the level of fractal surfaces, which, in their turn, can be described in terms of fractal distribution of conic points of positive and negative infinite curvatures. As a consequence, we have replaced velocity on a particular geodesic by fractal velocity field of the whole infinite ensemble of geodesics. This representation is similar to that of fluid mechanics where the motion of the fluid is described in terms of its velocity field, density, and pressure. We will, indeed, recover the fundamental equations of fluid mechanics (Euler and continuity equations), but we will write them in terms of a density of probability (as defined by the set of geodesics) instead of a density of matter and adding an additional term of quantum pressure (the expression of fractal geometry).

(iv) The local differential time invariance is broken, so the time-derivative of the fractal field $Q$ can be written twofold:

$$
\begin{aligned}
& \frac{d_{+} Q}{d t}=\lim _{\Delta t \rightarrow 0_{+}} \frac{Q(t+\Delta t)-Q(t)}{\Delta t}, \\
& \frac{d_{-} Q}{d t}=\lim _{\Delta t \rightarrow 0_{-}} \frac{Q(t)-Q(t-\Delta t)}{\Delta t} .
\end{aligned}
$$

Both definitions are equivalent in the differentiable case $d t \rightarrow-d t$. In the nondifferentiable situation, these definitions are no longer valid, since limits are not defined anymore. Fractal theory defines physics in relationship with the function behaviour during the "zoom" operation on the time resolution $\delta t$, which is here identified with the differential element $d t$ (substitution principle), which is considered an independent variable. The standard field $Q(t)$ is therefore replaced by fractal field $Q(t, d t)$, explicitly dependent on time resolution interval, whose derivative is not defined at the unnoticeable limit $d t \rightarrow 0$. As a consequence, this leads to the two derivatives of the fractal field $Q$ as explicit functions of the two variables $t$ and $d t$

$$
\begin{aligned}
& \frac{d_{+} Q}{d t}=\lim _{\Delta t \rightarrow 0_{+}} \frac{Q(t+\Delta t, \Delta t)-Q(t, \Delta t)}{\Delta t}, \\
& \frac{d_{-} Q}{d t}=\lim _{\Delta t \rightarrow 0_{-}} \frac{Q(t, \Delta t)-Q(t-\Delta t, \Delta t)}{\Delta t} .
\end{aligned}
$$

Notation "+" corresponds to the forward process, while "_" to the backward one.

Let us particularize (A.3) for the spatial coordinates. $t$ results in

$$
d_{ \pm} \mathbf{X}=d_{ \pm} \mathbf{x}+d_{ \pm} \xi
$$

Since, according to $[12-14,19-22]$, we can write

$$
\left\langle d_{ \pm} X^{i}\right\rangle=d_{ \pm} x^{i}
$$

from (A.4) by averaging, it results in

$$
\left\langle d \xi_{ \pm}^{i}\right\rangle=0
$$

(v) The differential fractal part satisfies the fractal equation:

$$
d_{ \pm} \xi^{i}=\lambda_{ \pm}^{i}\left(\frac{d t}{\tau}\right)^{1 / D_{F}}
$$

where $\lambda_{ \pm}^{i}$ are some constant coefficients, $d t$ is the time differential, $\tau$ is the reference time scale, and $D_{F}$ is a constant fractal dimension. We note that the use of any Kolmogorov or Hausdorff definition [12-14, 19-22] can be accepted for fractal 
dimension, but once a certain definition is admitted, it should be used until the end of analyzed dynamics.

(vi) The local differential time reflection invariance is recovered by combining the two derivatives, $d_{+} / d t$ and $d_{-} / d t$, in the complex operator:

$$
\frac{\widehat{d}}{d t}=\frac{1}{2}\left(\frac{d_{+}+d_{-}}{d t}\right)-\frac{i}{2}\left(\frac{d_{+}-d_{-}}{d t}\right) .
$$

Applying this operator to the "position vector," a complex velocity yields

$$
\begin{aligned}
\widehat{\mathbf{V}} & =\frac{\widehat{d} \mathbf{X}}{d t}=\frac{1}{2}\left(\frac{d_{+} \mathbf{X}+d_{-} \mathbf{X}}{d t}\right)-\frac{i}{2}\left(\frac{d_{+} \mathbf{X}-d_{-} \mathbf{X}}{d t}\right) \\
& =\frac{\mathbf{V}_{+}+\mathbf{V}_{-}}{2}-i \frac{\mathbf{V}_{+}-\mathbf{V}_{-}}{2}=\mathbf{V}_{D}-i \mathbf{V}_{F}
\end{aligned}
$$

with

$$
\begin{aligned}
& \mathbf{V}_{D}=\frac{\mathbf{V}_{+}+\mathbf{V}_{-}}{2}, \\
& \mathbf{V}_{F}=\frac{\mathbf{V}_{+}-\mathbf{V}_{-}}{2} .
\end{aligned}
$$

The real part, $\mathbf{V}_{D}$, of the complex velocity $\widehat{\mathbf{V}}$ represents the standard classical velocity, which does not depend on resolution, while the imaginary part, $\mathbf{V}_{F}$, is a new quantity coming from fractality.

\section{B. Covariant Derivative}

Let us now assume that curves describing movements of complex systems (continuous but nondifferentiable) are immersed in a 3-dimensional space and that $\mathbf{X}$ of components $X^{i}(i=\overline{1,3})$ is the position vector of a point on the curve. Let us also consider the fractal field $Q(\mathbf{X}, t)$ and expand its total differential up to the second order:

$$
d_{ \pm} Q=\frac{\partial Q}{\partial t} d t+\nabla Q \cdot d_{ \pm} \mathbf{X}+\frac{1}{2} \frac{\partial^{2} Q}{\partial X^{i} \partial X^{j}} d_{ \pm} X^{i} d_{ \pm} X^{j} .
$$

Relations (B.1) are valid in any point both for the spatial manifold and for the points $\mathbf{X}$ on the fractal curve selected in relations (B.1). Hence, the forward and backward average values of these relations take the form

$$
\begin{aligned}
\left\langle d_{ \pm} Q\right\rangle= & \left\langle\frac{\partial Q}{\partial t} d t\right\rangle+\left\langle\nabla Q \cdot d_{ \pm} \mathbf{X}\right\rangle \\
& +\frac{1}{2}\left\langle\frac{\partial^{2} Q}{\partial X^{i} \partial X^{j}} d_{ \pm} X^{i} d_{ \pm} X^{j}\right\rangle .
\end{aligned}
$$

The following aspects should be mentioned: the mean value of function $Q$ and its derivatives coincide with themselves, and the differentials $d_{ \pm} X^{i}$ and $d t$ are independent; therefore, the average of their products coincides with the product of averages. Consequently, (B.2) becomes

$$
d_{ \pm} Q=\frac{\partial Q}{\partial t} d t+\nabla Q\left\langle d_{ \pm} \mathbf{X}\right\rangle+\frac{1}{2} \frac{\partial^{2} Q}{\partial X^{i} \partial X^{j}}\left\langle d_{ \pm} X^{i} d_{ \pm} X^{j}\right\rangle
$$

or more, using (A.4) and (A.6),

$$
\begin{aligned}
d_{ \pm} Q= & \frac{\partial Q}{\partial t} d t+\nabla Q \cdot d_{ \pm} \mathbf{X}+\frac{1}{2} \frac{\partial^{2} Q}{\partial X^{i} \partial X^{j}} \\
& \times\left(d_{ \pm} X^{i} d_{ \pm} X^{j}+\left\langle d_{ \pm} \xi^{i} d_{ \pm} \xi^{j}\right\rangle\right) .
\end{aligned}
$$

Even if the average value of the fractal coordinate $d_{ \pm} \xi^{i}$ is null (see (A.6)), for higher order of fractal coordinate average, the situation can still be different. Let us focus on the averages $\left\langle d_{ \pm} \xi^{i} d_{ \pm} \xi^{j}\right\rangle$. If $i \neq j$, these averages are zero due to the independence of $d_{ \pm} \xi^{i}$ and $d_{ \pm} \xi^{j}$. So, using (A.7), we can write

$$
\left\langle d_{ \pm} \xi^{i} d_{ \pm} \xi^{j}\right\rangle=\lambda_{ \pm}^{i} \lambda_{ \pm}^{j}\left(\frac{d t}{\tau}\right)^{\left(2 / D_{F}\right)-1}\left(\frac{d t}{\tau}\right) .
$$

Then, (B.4) may be written as follows:

$$
\begin{aligned}
d_{ \pm} Q= & \frac{\partial Q}{\partial t} d t+d_{ \pm} \mathbf{x} \cdot \nabla Q+\frac{1}{2} \frac{\partial^{2} Q}{\partial X^{i} \partial X^{j}} d_{ \pm} x^{i} d_{ \pm} x^{j} \\
& +\frac{1}{2} \frac{\partial^{2} Q}{\partial X^{i} \partial X^{j}} \lambda_{+}^{i} \lambda_{+}^{j}\left(\frac{d t}{\tau}\right)^{\left(2 / D_{F}\right)-1}\left(\frac{d t}{\tau}\right) .
\end{aligned}
$$

If we divide by $d t$ and neglect the terms containing differential factors, (B.6) is reduced to

$$
\frac{d_{ \pm} Q}{d t}=\frac{\partial Q}{\partial t}+\mathbf{V}_{ \pm} \nabla Q+\frac{1}{2} \frac{\partial^{2} Q}{\partial X^{i} \partial X^{j}} \frac{\lambda_{-}^{i} \lambda_{-}^{j}}{\tau}\left(\frac{d t}{\tau}\right)^{\left(2 / D_{F}\right)-1}
$$

These relations also allow us to define the operators:

$$
\frac{d_{ \pm}}{d t}=\frac{\partial}{\partial t}+\mathbf{V}_{ \pm} \cdot \nabla+\frac{\lambda_{+}^{i} \lambda_{+}^{j}}{2 \tau}\left(\frac{d t}{\tau}\right)^{\left(2 / D_{F}\right)-1} \frac{\partial^{2}}{\partial X^{i} \partial X^{j}} .
$$

Under these circumstances, let us calculate $\widehat{d} \mathrm{Q} / d t$. Taking into account (B.8), (A.8), and (A.9), we will obtain

$$
\begin{aligned}
\frac{\widehat{d} Q}{d t}= & \frac{1}{2}\left[\frac{d_{+} Q}{d t}+\frac{d_{-} Q}{d t}-i\left(\frac{d_{+} Q}{d t}-\frac{d_{-} Q}{d t}\right)\right] \\
= & \frac{\partial Q}{\partial t}+\widehat{\mathbf{V}} \cdot \nabla+\frac{(d t / \tau)^{\left(2 / D_{F}\right)-1}}{4 \tau} \\
& \cdot\left[\left(\lambda_{+}^{i} \lambda_{+}^{j}+\lambda_{-}^{i} \lambda_{-}^{j}\right)-i\left(\lambda_{+}^{i} \lambda_{+}^{j}-\lambda_{-}^{i} \lambda_{-}^{j}\right)\right] \frac{\partial^{2} Q}{\partial X^{i} \partial X^{j}} .
\end{aligned}
$$

This relation also allows us to define the fractal operator:

$$
\begin{aligned}
\frac{\widehat{d}}{d t}= & \frac{\partial}{\partial t}+\widehat{\mathbf{V}} \cdot \nabla+\frac{(d t / \tau)^{\left(2 / D_{F}\right)-1}}{4 \tau} \\
& \cdot\left[\left(\lambda_{+}^{i} \lambda_{+}^{j}+\lambda_{-}^{i} \lambda_{-}^{j}\right)-i\left(\lambda_{+}^{i} \lambda_{+}^{j}-\lambda_{-}^{i} \lambda_{-}^{j}\right)\right] \frac{\partial^{2} Q}{\partial X^{i} \partial X^{j}}
\end{aligned}
$$

Particularly, by choosing

$$
\lambda_{+}^{i} \lambda_{+}^{j}=-\lambda_{-}^{i} \lambda_{-}^{j}=2 \lambda^{2} \delta^{i j},
$$

where $\lambda$ is the reference length scale, the fractal operator (B.10) (covariant derivative) takes the usual form:

$$
\frac{\widehat{d}}{d t}=\frac{\partial}{\partial t}+\widehat{\mathbf{V}} \cdot \nabla-i \frac{\lambda^{2}}{\tau}\left(\frac{d t}{\tau}\right)^{\left(2 / D_{F}\right)-1} \Delta .
$$




\section{References}

[1] B.-Y. Yaneer, Dynamics of Complex Systems, The Advanced Book Program, Addison-Wesley, Reading, Mass, USA, 1997.

[2] S. V. Buldyrev, R. Parshani, G. Paul, H. E. Stanley, and S. Havlin, "Catastrophic cascade of failures in interdependent networks," Nature, vol. 464, no. 7291, pp. 1025-1028, 2010.

[3] K. Kaneko, Complex Systems: Chaos and Beyond, A Constructive Approach with Applications in Life Sciences, 2001.

[4] C. Gros, Complex and Adaptative Dynamical Systems: A Primer, 2011.

[5] R. Badii and A. Politi, Complexity: Hierarchical Structure and Scaling in Physics, Cambridge University Press, Cambridge, UK, 1997.

[6] M. Mitchell, Complexity: A Guided Tour, Oxford University Press, Oxford, UK, 2009.

[7] G. W. Flake, The Computational Beauty of Nature, The MIT Press, Cambridge, Mass, USA, 1998.

[8] A. T. Winfree, The Geometry of Biological Time, Springer, New York, NY, USA, 2nd edition, 2000.

[9] P. Davies, The New Physics, Cambridge University Press, 1992.

[10] C. H. Bennett, "How to define complexity in physics, and why," in Complexity, Entropy, and the Physics of Information, W. H. Zurek, Ed., pp. 443-454, Addison-Wesley, Reading, Mass, USA, 1990.

[11] Mandelbrot and B. Benoit, The Fractal Geometry of Nature, W. H. Freeman, New York, NY, USA, 1983.

[12] L. Nottale, Fractal Space-Time and Microphysics: Towards a Theory of Scale Relativity, World Scientific, Singapore, 1993.

[13] L. Nottale, Scale Relativity and Fractal Space-Time-A New Approach To Unifying Relativity and Quantum Mechanics, Imperial College Press, London, UK, 2011.

[14] L. Nottale, "Fractals and the quantum theory of spacetime," International Journal of Modern Physics A, vol. 4, pp. 5047-5117, 1989.

[15] G. M. Viswanathan, F. Bartumeus, S. V. Buldyrev et al., "Lévy flight random searches in biological phenomena," Physica A, vol. 314, no. 1-4, pp. 208-213, 2002.

[16] M. F. Shlesinger, J. Klafter, and G. Zumofen, "Above, below and beyond Brownian motion," American Journal of Physics, vol. 67, no. 12, pp. 1253-1259, 1999.

[17] M. S. El Naschie and O. E. Rossler, "Quantum mechanics and chaotic fractals," Chaos, Solitons and Fractals, vol. 4, no. 3, pp. 307-309, 1994.

[18] P. Weibel, G. Ord, and O. E. Rosler, Space Time Physics and Fractility, Springer, New York, NY, USA, 2005.

[19] S. Gurlui, M. Agop, P. Nica, M. Ziskind, and C. Focşa, "Experimental and theoretical investigations of transitory phenomena in high-fluence laser ablation plasma," Physical Review E, vol. 78, Article ID 026405, 2008.

[20] M. Agop, D. G. Dimitriu, O. Niculescu, E. Poll, and V. Radu, "Experimental and theoretical evidence for the chaotic dynamics of complex structures," Physica Scripta, vol. 87, no. 4, Article ID 045501, 2013.

[21] M. Agop, N. Forna, I. Casian-Botez, and C. Bejenariu, "New theoretical approach of the physical processes in nanostructures," Journal of Computational and Theoretical Nanoscience, vol. 5, no. 4, pp. 483-489, 2008.

[22] I. Casian-Botez, M. Agop, P. Nica, V. Paun, and G. V. Munceleanu, "Conductive and convective types behaviors at nano-time scales," Journal of Computational and Theoretical Nanoscience, vol. 7, no. 11, pp. 2271-2280, 2010.

[23] M. Agop, P. E. Nica, S. Gurlui, C. Focşa, V. P. Păun, and M. Colotin, "Implications of an extended fractal hydrodynamic model," The European Physical Journal D, vol. 56, no. 3, pp. 405$419,2010$.

[24] E. M. Lifshiëtìs and L. D. Landau, Fluid Mechanics, Pergamon Press, 1987.

[25] G. V. Munceleanu, V.-P. Paun, I. Casian-Botez, and M. Agop, "The microscopic-macroscopic scale transformation through a chaos scenario in the fractal space-time theory," International Journal of Bifurcation and Chaos, vol. 21, no. 2, pp. 603-618, 2011.

[26] P. Nica, M. Agop, S. Gurlui, C. Bejinariu, and C. Focsa, "Characterization of aluminum laser produced plasma by target current measurements," Japanese Journal of Applied Physics, vol. 51, Article ID 106102, 10 pages, 2012.

[27] P. Nica, P. Vizureanu, M. Agop et al., "Experimental and theoretical aspects of aluminum expanding laser plasma," Japanese Journal of Applied Physics, vol. 48, no. 6, Article ID 066001, 2009.

[28] O. C. Zienkievicz and R. L. Taylor, The Finite Element Method, McGraw-Hill, New York, NY, USA, 1991.

[29] C. Ursu, O. G. Pompilian, S. Gurlui et al., " $\mathrm{Al}_{2} \mathrm{O}_{3}$ ceramics under high-fluence irradiation: plasma plume dynamics through space- and time-resolved optical emission spectroscopy," Applied Physics A, vol. 101, no. 1, pp. 153-159, 2010.

[30] O. G. Pompilian, S. Gurlui, P. Nemec, V. Nazabal, M. Ziskind, and C. Focşa, "Plasma diagnostics in pulsed laser deposition of GaLaS chalcogenides," Applied Surface Science, vol. 278, pp. 352-356, 2013.

[31] C. Focsa, P. Nemec, M. Ziskind, C. Ursu, S. Gurlui, and V. Nazabal, "Laser ablation of $\mathrm{As}_{x} \mathrm{Se}_{100-x}$ chalcogenide glasses: plume investigations," Applied Surface Science, vol. 255, no. 10, pp. 5307-5311, 2009.

[32] C. Ursu, S. Gurlui, C. Focsa, and G. Popa, "Space- and timeresolved optical diagnosis for the study of laser ablation plasma dynamics," Nuclear Instruments and Methods in Physics Research $B$, vol. 267, no. 2, pp. 446-450, 2009.

[33] S. Gurlui and C. Focşa, "Laser ablation transient plasma structures expansion in vacuum," IEEE Transactions on Plasma Science, vol. 39, no. 11, pp. 2820-2821, 2011.

[34] D. L. Aronstein and C. R. Stround Jr., "Fractional wave-function revivals in the infinite square well," Physical Review A, vol. 55, no. 6, pp. 1050-2947, 1997.

[35] C. P. Cristescu, Dinamici Neliniare Si Haos, Fundamente Teoretice Si Aplicatii, Academiei Romane, Bucuresti, Romania, 2008.

[36] J. Zhang and W. Tang, "Optimal control for a class of chaotic systems," Journal of Applied Mathematics, vol. 2012, Article ID 859542, 20 pages, 2012.

[37] J. B. Polly and J. M. McDonough, "Application of the poor Man's navier-stokes equations to real-time control of fluid flow," Journal of Applied Mathematics, vol. 2012, Article ID 746752, 18 pages, 2012.

[38] E. S. Bacaita, C. Bejinariu, B. Zoltan et al., "Nonlinearities in drug release process from polymeric microparticles: longtime-scale behaviour," Journal of Applied Mathematics, vol. 2012, Article ID 653720, 26 pages, 2012.

[39] K. Yuan, J. Cao, and S. Fei, "Synchronization of coupled networks with mixed delays by intermittent control," Journal of Applied Mathematics, vol. 2012, Article ID 927609, 13 pages, 2012. 


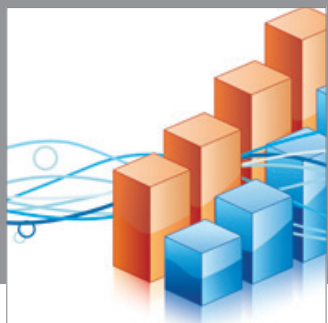

Advances in

Operations Research

mansans

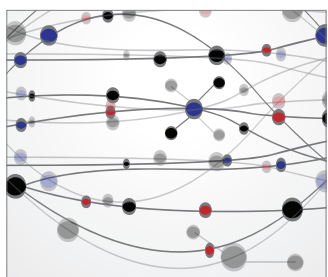

The Scientific World Journal
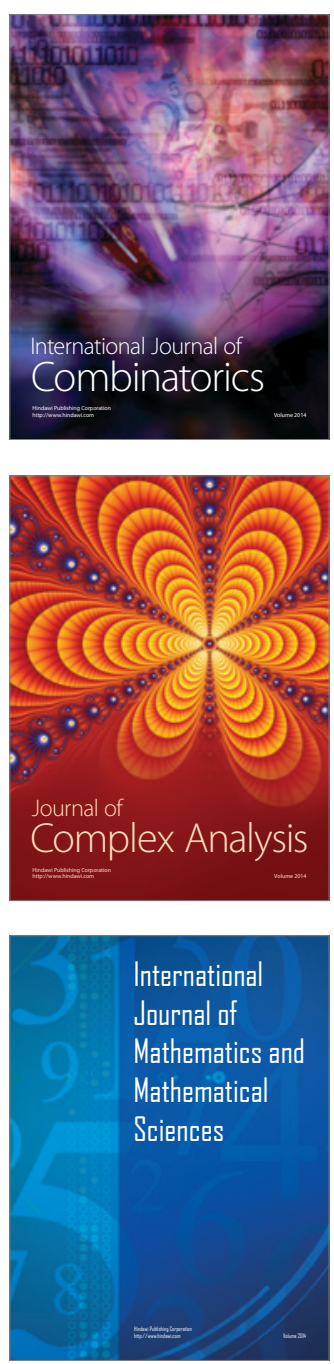
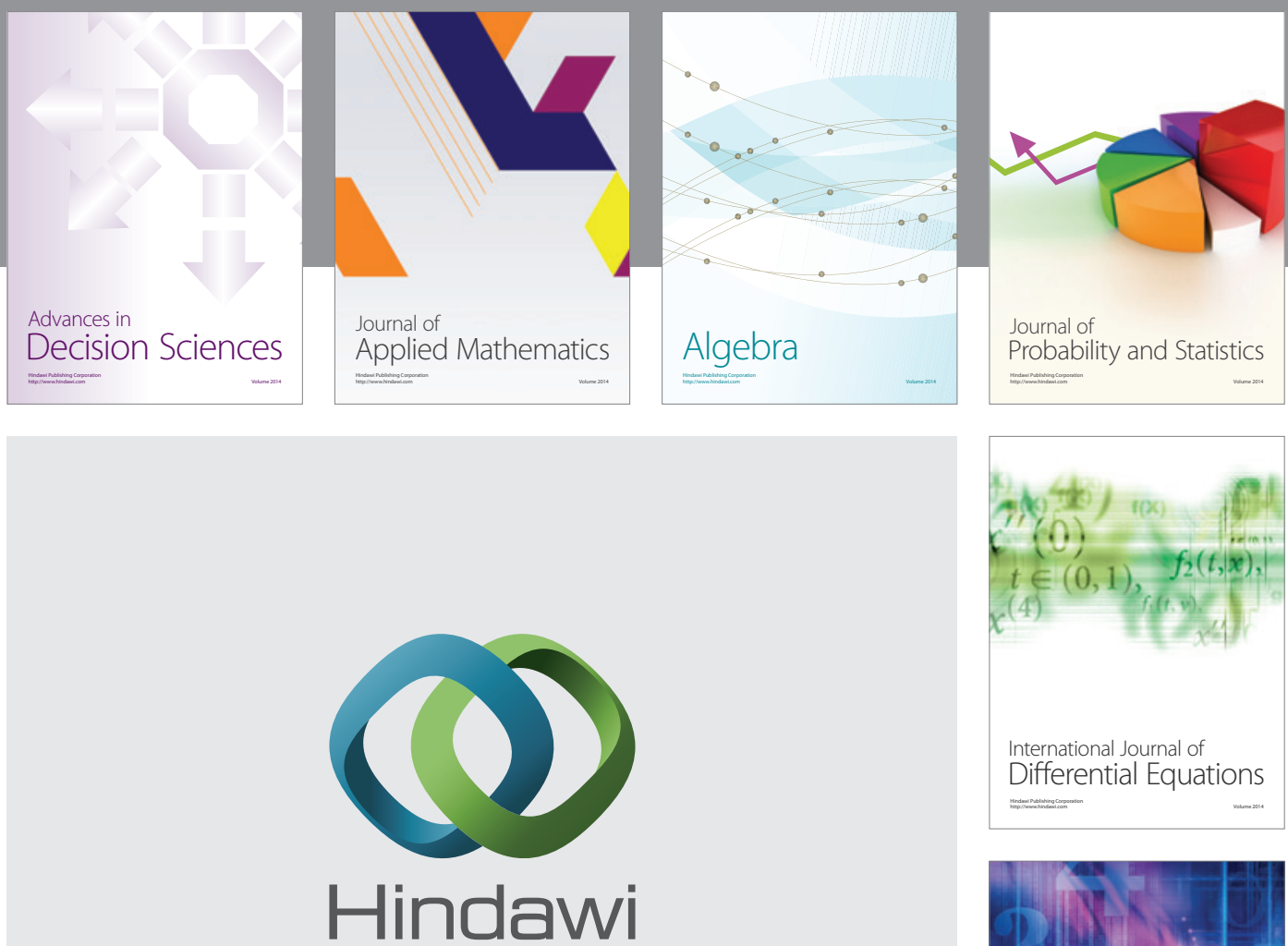

Submit your manuscripts at http://www.hindawi.com
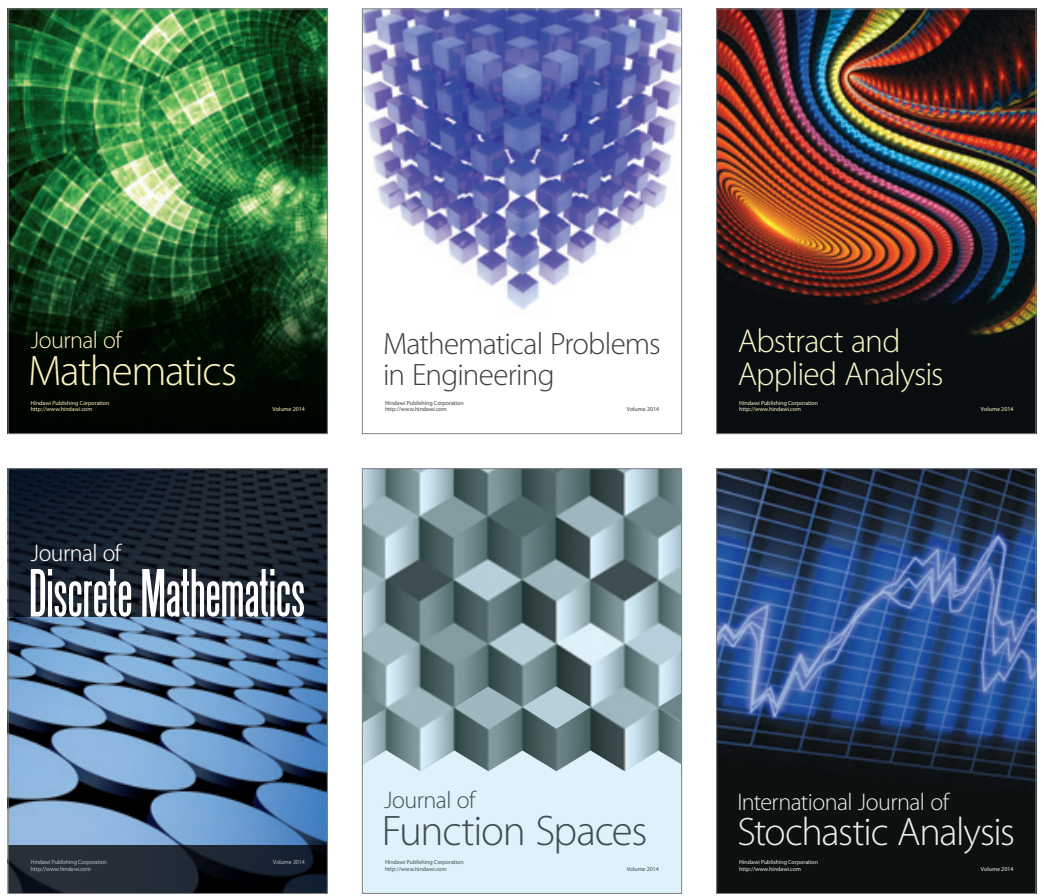

Journal of

Function Spaces

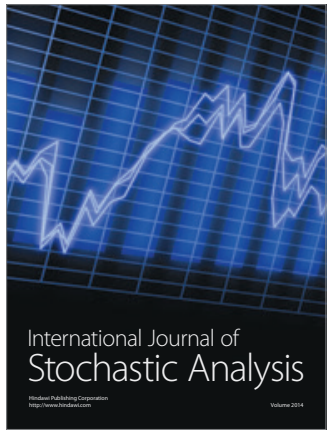

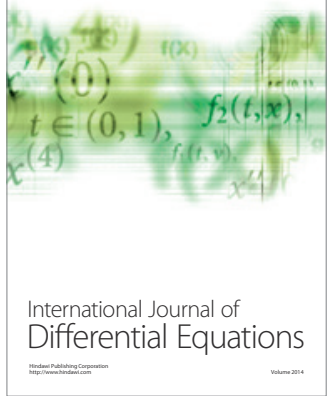
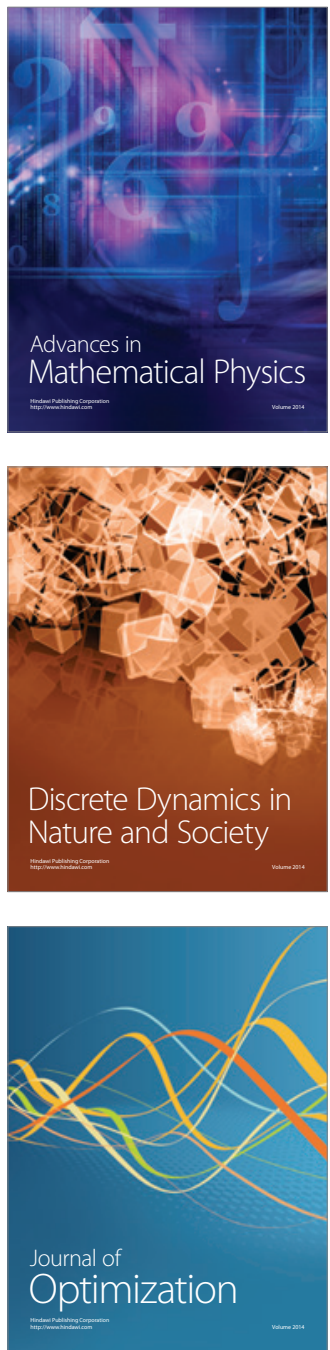